\title{
Stellar cusp and warm dust at the heart of NGC 1068 ${ }^{\star \star \star}$
}

\author{
D. Rouan, L. Grosset, and D. Gratadour
}

\begin{abstract}
LESIA, Observatoire de Paris, PSL Research University, CNRS, Sorbonne Universités, UPMC Univ. Paris 06, Univ. Paris Diderot, Sorbonne Paris Cité, 5 place Jules Janssen, 92190 Meudon, France e-mail: daniel.rouan@obspm.fr
\end{abstract}

Received 24 October 2018 / Accepted 5 March 2019

\begin{abstract}
Aims. Establishing precisely how stars and interstellar medium distribute within the central $100 \mathrm{pc}$ area around an AGN, down to the pc scale, is key to understanding the late stages of transfer of matter onto the accretion disc.

Methods. Using adaptive optics-assisted (SPHERE-VLT) near-IR images in the $H$ band, $K_{s}$ band, and several narrow bands of the Seyfert 2 galaxy NGC 1068, we analysed the radial distribution of brightness in the central $r<100 \mathrm{pc}$ area down to the pc scale. The median-averaged radial profiles are fitted by a cusp (power law) plus a central point source. A simple radiative transfer model is also used to interpret the data.

Results. We find that the fit of radial brightness profiles beyond $10 \mathrm{pc}$ is done quite precisely at $K_{s}$ band by a cusp of exponent -2.0 plus a central point source and by a cusp of exponent -1.2 at $H$. The difference of exponents between $H$ band and $K_{s}$ band can be explained by differential extinction, provided that the distribution of dust is itself cuspy, with an exponent -1.0 . However, the derived stellar density is found to follow a $r^{-4}$ cusp, which is much steeper than any other cusp, either theoretically predicted around a massive black hole, or observed in the centre of early- and late-type galaxies or in mergers. Introducing a segregation in the stellar population with a central excess of giant stars leads to a somewhat less steep exponent; however, the de-reddened luminosity of the stellar cusp, as well as the mass of dust and gas all appear much too high to be realistic. An alternative scenario, where the $K_{s}$-band profile is well fitted by a combination of radiation from a stellar cusp identical to the $H$-band profile and thermal emission of warm/hot dust heated by the central engine appears much more satisfactory. NGC 1068 is shown to satisfy a relationship between half-light radius, cusp luminosity, and exponent that we established using a sample of luminous infrared galaxies (LIRGs) and ultraluminous infrared galaxies ULIRGs. This suggests that the cusp is the remnant of a recent starburst. We identify the central point-like source with the very hot dust at the internal wall of the putative torus and derive an intrinsic luminosity that requires a central extinction $A_{K} \approx 8$, a value consistent with predictions by several torus models.

Conclusions. The overall picture revealed by this study is closely consistent with the scheme of a central rather steep stellar cusp, embedded in a diluted medium of warm dust, while a compact and dense structure identified with the putative torus is required to interpret a highly reddened point-like central source of very hot dust.
\end{abstract}

Key words. instrumentation: adaptive optics - galaxies: active - dust, extinction - galaxies: Seyfert

\section{Introduction}

In an active galactic nucleus (AGN), considered here as the very central region encompassing the stellar cluster and a dense molecular core, the feeding of the central engine (hereafter $\mathrm{CE}$ ), i.e. the accretion disc, requires a matter accretion rate that ranges from 0.3 in Seyfert galaxies (Bian \& Zhao 2003) to $10 M_{\odot} \mathrm{yr}^{-1}$ (Hopkins et al. 2012) for the most luminous quasars. How the very final transfer of matter is achieved from kpc scale to the subparsec size of the accretion disc is still a matter of debate since the mechanism of loss of angular momentum at scales below $100 \mathrm{pc}$ has not been clearly established (see Goodman 2003 for a review), nor is there any clear relationship between this feeding mechanism and the likely existence of an obscuring torus, the paradigm proposed by Antonucci \& Miller (1985) years ago to interpret the Seyfert $1 / 2$ duality, and that still remains valid in

\footnotetext{
* The reduced images are only available at the CDS via anonymous ftp to cdsarc.u-strasbg.fr $(130.79 .128 .5)$ or via http: //cdsarc.u-strasbg.fr/viz-bin/qcat?J/A+A/625/A100

$\star \star$ Based on observations collected at the European Southern Observatory, Paranal, Chile, through observing proposals 60.A-9361(A) and 097.B-0840(A).
}

the light of recent ALMA or SPHERE polaro-imaging observations (García-Burillo et al. 2016; Gratadour et al. 2015). One of the most difficult problems for hydrodynamical models is to take into account a huge range of scales, from $10 \mathrm{kpc}$ in spiral arms down to $0.1 \mathrm{pc}$ in the accretion disc while integrating all the possible processes such as cooling, star formation, and self-gravity of the gas, which are all highly non-linear.

Hopkins et al. (2012), using results from their multiscale hydrodynamical model (Hopkins \& Quataert 2010, 2011), recently proposed that the formation, through instabilities, of lopsided eccentric gas plus stellar discs about $10 \mathrm{pc}$ in size would be the dominant cause for angular momentum transport below this scale. The asymmetry of the stellar disc would be the main cause of the strong torque exerted on the gas. The model also predicts that surface densities of gas and stars both follow cuspy distributions.

Performing observations in the visible to IR that could test the validity of this proposal, especially evidencing the stellar disc, is not obvious because there are several limits: i) we are dealing with scales that are largely subarcsec $(1 \mathrm{pc}=20$ mas at $10 \mathrm{Mpc})$; ii) the central region is very obscured by the huge concentration of dust; and iii) the bright dazzling AGN totally 
dominates the flux, making it difficult to detect fainter structures associated with warm dust emission or stellar population in the immediate neighbourhood. Of course millimetric interferometry, especially using ALMA, could solve the two first issues, but it is not suited to probe stellar emission. On the other hand, the combination of adaptive optics (AO) on large telescopes and imaging in the near-IR (NIR) gives us the opportunity to tackle the problem, at least on nearby AGNs. This is the goal of this paper where we present an analysis of adaptive optics images of NGC 1068 in $K_{s}$ and $H$ bands, and in several narrow-band filters, obtained with SPHERE on the VLT.

NGC 1068 is a Seyfert 2 galaxy that has been the target for many studies about AGN since it is one of the closest active nuclei (14.4 Mpc, following Bland-Hawthorn et al. 1997) and therefore one of the brightest. One consequence is that the nucleus is bright enough to be used as the guide source for the wavefront sensor of the AO system, allowing us to obtain high angular resolution images in the NIR, for example with PUEO-CFHT (Gratadour et al. 2003), NAOS-VLT (Rouan et al. 2004; Gratadour et al. 2006; Prieto et al. 2005), SINFONI-VLT (Davies et al. 2007; Müller Sánchez et al. 2009), or more recently using SPHERE-VLT (Gratadour et al. 2015).

The later observations were conducted during the SPHERE Science Verification (SV) programme in December 2014. The $H$ - and $K_{s}$-band broad-band images, showed a polarisation angle map with a clear centro-symmetric pattern, tracing both parts of the ionisation bicone. More importantly, they featured a central non-centro-symmetric pattern approximately $60 \mathrm{pc} \times 20 \mathrm{pc}$ wide, with aligned polarisation, that was interpreted as the trace of the outer envelope of the torus, revealed through a double scattering process (Gratadour et al. 2015; Grosset et al. 2018). It is this set of images, complemented by images obtained in narrowband filters in September 2016, again with SPHERE, that we re-processed, but without using the polarisation information. In this paper we concentrate on the radial distribution of brightness.

The paper is organised as follows. The data processing and radial profile fitting is presented in Sect. 2. The discussion on the power-law radial profiles at $H$ and $K_{s}$ band and their interpretation in terms of a reddened cuspy stellar distributions through a simple transfer model is presented in Sect. 3 after some discussion about various classes of cusps observed in the centre of galaxies. In Sect. 4 we discuss an alternative and more acceptable model where warm/hot dust heated by the central engine is mixed to stellar emission. Finally, we examine the central pointlike source in Sect. 5 as well as the constraints brought on the dust opacity by its luminosity, before concluding in Sect. 6 .

\section{Data set and data processing}

The $K_{s^{-}}$and $H$-band images obtained with SPHERE in December 2014 are shown in Fig. 1, using a Asinh scaling law for the intensity that enhances the low flux levels. Details about observations and data reduction have been published in Gratadour et al. (2015). The pixel size is 12.25 mas, corresponding to $0.855 \mathrm{pc}$ assuming a distance of $14.4 \mathrm{Mpc}$. The field of view is $6.27 \times 6.27 \mathrm{arcsec}^{2}$, or $438 \times 438 \mathrm{pc}^{2}$, and the angular resolution, as measured on the point spread function (PSF), is 58 mas and 53 mas in $K_{s}$ and $H$, respectively.

In the $H$-band image can be seen the elongated disc, slightly asymmetric with respect to the $\mathrm{CE}$, well defined by a strong peak. The disc size is about $135 \mathrm{pc} \times 180 \mathrm{pc}$, choosing as the limit the distance where the slope of the radial distribution flattens. Assuming that the corresponding iso-contour delineates the disc, the barycentre of this contour is offset by 6 pixels $(5.1 \mathrm{pc})$
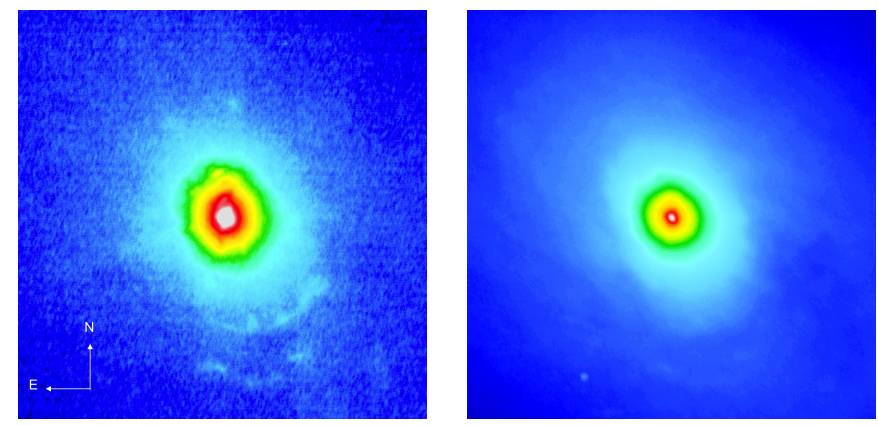

Fig. 1. $K_{s}$-band (left) and $H$-band (right) images of the central $438 \times 438 \mathrm{pc}$ of NGC 1068 , obtained with SPHERE, where 1 arcsec is $70 \mathrm{pc}$. The intensity scaling corresponds to an Asinh law. For the $K_{s}$ band image the flux at peak (white area) is $2.3 \times 10^{-3} \mathrm{Jy} \mathrm{pixel}^{-1}$, and in the light blue area it is $\approx 6.0 \times 10^{-6} \mathrm{Jy} \mathrm{pixel}^{-1}$. For the $H$-band image the flux at peak (white) is $2.0 \times 10^{-4} \mathrm{Jy} \mathrm{pixel}^{-1}$, and in the light blue area it is $\approx 7.5 \times 10^{-6} \mathrm{Jy} \mathrm{pixel}^{-1}$.

to the west and 1 pixel $(0.9 \mathrm{pc})$ to the north with respect to the central peak. In the $K_{s}$-band image, the asymmetry is less pronounced and, for instance, the centre of gravity of the contour delineating about the same area is only shifted by 2.5 pixels $(2.1 \mathrm{pc})$ and 1.5 pixels $(1.3 \mathrm{pc})$ respectively to the west and north.

We should now ask an important question: what is the source of emission at $H$ band and $K_{s}$ band? The answer is not trivial, especially concerning the $K_{S}$ band. We expect an important contribution from stellar emission, but we also expect a significant contribution from very hot dust, close to sublimation temperature, arising from the internal radius of the likely torus. For instance, Gratadour et al. (2003) showed clearly that the steeper slope of the NIR spectrum when approaching the central peak is nicely explained by this very hot dust. In the following, our main concern is to discriminate between the respective contributions of the two sources of radiation.

Let us first examine the medium scale. We present $\mathrm{f}$ in Fig. 2 the median brightness profile in $H$ band extending up to $430 \mathrm{pc}$, i.e. well beyond the molecular ring of $r \approx 200 \mathrm{pc}$ detected by Müller Sánchez et al. (2009) and confirmed by ALMA (García-Burillo et al. 2014). In the same figure, two fits are represented: a power-law fit for the most internal part $(r \leq 110 \mathrm{pc})$ and an exponential fit for the external part (up to $r=430 \mathrm{pc}$ ). Both fits are fairly good and, assuming that it is essentially the stellar component seen at $H$, we conclude that when going inward, a disc distribution (here of scale length $R_{\mathrm{c}}=410 \mathrm{pc}$ ) is superseded by a cuspy (i.e. power-law) distribution of exponent 1.2 for $r \leq 110 \mathrm{pc}$.

In Fig. 3 we plot the median radial brightness profile around the central peak, measured in the $K_{s}$-band and $H$-band images, together with the PSF profile at $K_{s}$ band measured on the nearby reference star BD-00413 whose visible magnitude is close to that of the nucleus of NGC 1068, so that the AO performances can be considered very similar. Two features should be noted. First, the $H$-band profile is almost perfectly fitted by a power law of exponent -1.2 ; we come back to the meaning of this behaviour in the next section. Second, the $K_{s}$-band profile wings, beyond a radius of about $17 \mathrm{pc}$, are also well fitted by a power law, but of exponent -2.0 . Interestingly, a fairly good fit to the whole data set is provided by a combination of this power-law profile truncated at $r<4$ pc plus the PSF profile properly weighted. This appears as the blue curve in Fig. 3 superimposed on the $K_{s}$-band actual profile. In particular, we note that the small bumps on the $K_{s}$-band profile, close to the peak, are well fitted by the first and even 


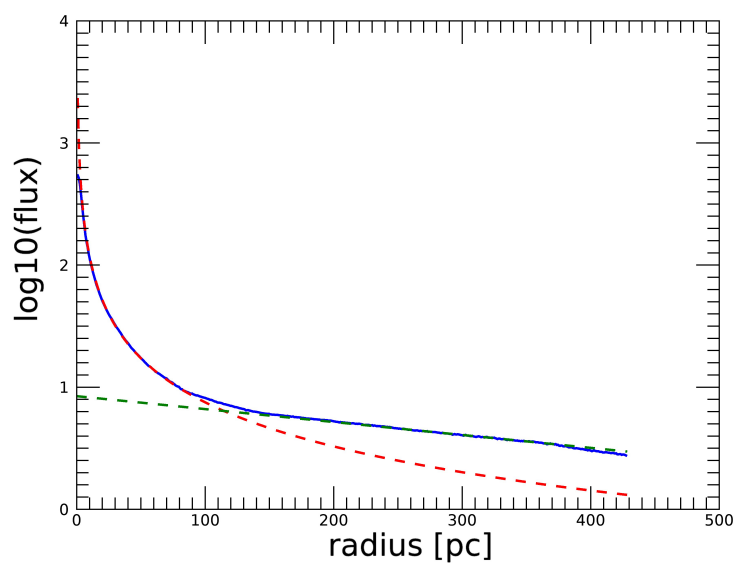

Fig. 2. Median radial brightness profile around the central peak, measured in the $H$-band. Blue solid line: Brightness profile in $H$ band up to $430 \mathrm{pc}$, estimated by performing a median of the pixels on successive circles centred on the central peak. Red dashed line: Power-law profile $r^{-1.2}$ fitted on the central distribution. Green dashed line: exponential profile fitted on the external observed distribution.

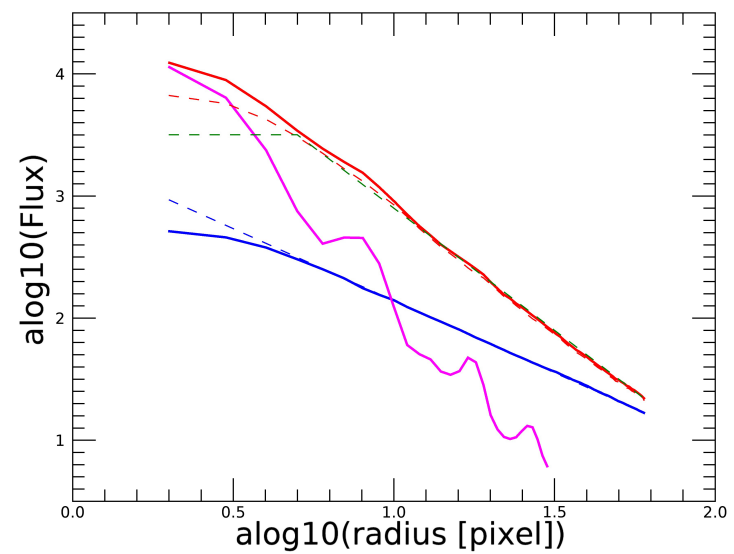

Fig. 3. Median radial brightness profile around the central peak. Solid red: measured at $K_{s}$; purple: PSF at $K_{s}$; red dash: difference between $K_{s}$ band and weighted PSF; green: truncated power-law profile in $r^{-2}$; solid blue: $H$-band profile; blue dash: power-law profile in $r^{-1.2}$.

the second Airy rings that clearly appear on the PSF profile. The straightforward interpretation of this fit is that the $K_{s}$-band profile results from the contribution of a central point source and of a smoother distribution that is directly related to the $H$-band emission. We tested other combinations by convolving the PSF with a Gaussian of various widths, but beyond a FWHM of 2 pixels $(1.7 \mathrm{pc})$ the fit degrades. The central source is thus practically point-like, and in any case its radius is not significantly larger than $0.8-1 \mathrm{pc}$.

In Sect. 5 we come back to the interpretation of this important result, namely that there is a quasi-unresolved central point source in $K_{S}$ band with no obvious counterpart in $H$. We concentrate in the next two sections on the power-law, or cuspy, component.

In Sept. 2016 we also obtained images with SPHERE in three narrow-band filters denoted cont $-H \quad(1.558 \mu \mathrm{m}$, $\Delta \lambda=0.0245 \mu \mathrm{m})$, cont $-K 1(2.103 \mu \mathrm{m}, \Delta \lambda=0.033 \mu \mathrm{m})$, and cont $-K 2(2.267 \mu \mathrm{m}, \Delta \lambda=0.034 \mu \mathrm{m})$. The details of the data reduction will be described in Grosset et al. (in prep.), a paper dedicated to the analysis of polarisation versus wavelength, but can also be found in Grosset (2017). The data processing is

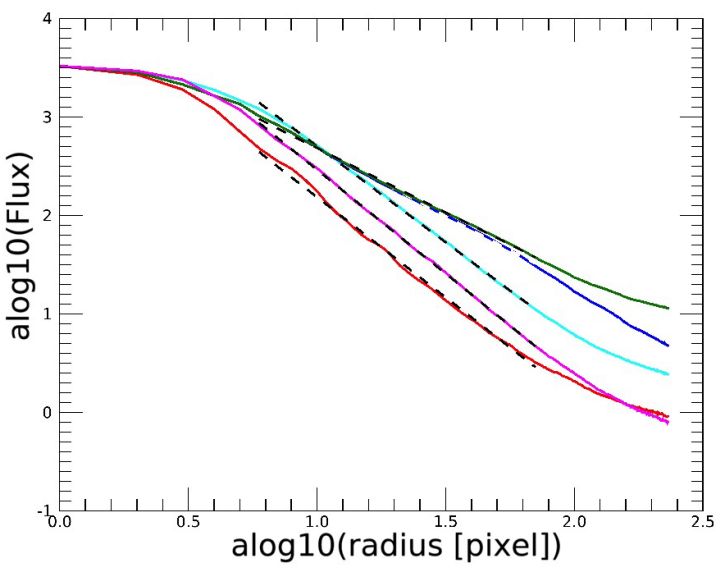

Fig. 4. Median radial brightness profile around the central peak for the cont $-H$ (blue), $H$-band (green), cont $-K 1$ (cyan), cont $-K 2$ (magenta), and $K_{s}$-band (red) images. The log-log scale shows a power-law behaviour for all four bands. The cont $-K 1$, cont $-K 2$, and $K_{s}$-band fluxes have been scaled on the $H$-band flux at the centre. The dashed line on each curve is the best linear fit for the range $5-60 \mathrm{pc}$.

essentially the same as that used for the $H$ - and $K_{s}$-band observations of December 2014.

We plot in Fig. 4 the median averaged profiles at the four wavelengths on a log-log scale. The following can be seen: i) the fairly linear behaviour of all profiles from 4 to 100 pixels ( 3.5 to $85 \mathrm{pc})$, as illustrated by the superimposed dash lines, and ii) the absolute value of the slope increasing with wavelength from $H$ band to $K_{s}$ band. The best fit power laws for the range 5-60 pc have exponents $-1.20,-1.36,-1.95,-2.11$, and -2.03 for $H$ band, cont $-H$, cont $-K 1$, cont $-K 2$, and $K_{s}$-band, respectively.

There are several possible origins for the power-law emission, and we examine the different cases in the following sections; however, the monotonic variation of the slope with respect to wavelength on a sample of five different filters is a strong indication that the cause of this variation is a continuously variable phenomenon rather than some spectral features such as emission lines. With this hypothesis in mind and taking into account that the signal-to-noise ratio is significantly lower in narrow-band images, we have chosen in the following to consider only the $H$-band and the $K_{s}$-band in our analysis; thanks to the broad range in wavelength coverage, any effect depending on wavelength will thus be clearly enhanced.

The first hypothesis, which could be well in line with the quite similar behaviour of the $H$-band and $K_{s}$-band radial brightness profiles and which corresponds to a situation often observed in the central region of galaxies (Böker 2008), is that in both bands we sense the emission from a stellar cusp, also called a nuclear stellar cluster. The second case, which is generally invoked in the case of NGC 1068 (Thatte et al. 1997; Gratadour et al. 2003) and other AGNs (Leja et al. 2018), is that there is warm to hot dust, heated by the $\mathrm{CE}$ and radiating in the infrared, especially at $K_{s}$. Of course, some mixing of this dust thermal radiation with the photospheric emission from a stellar cluster is likely, especially at $H$.

\section{A reddened stellar cusp?}

To start, we can assume that the $H$-band power-law profile is largely due to the stellar emission. First, because of this quasiperfect power-law behaviour, which is very similar to what is observed in many nuclei of galaxies (see next section), while 
it could hardly be explained by dust emission extending so far from the $\mathrm{CE}$, and second, because previous spectroscopic observations by Thatte et al. (1997) clearly identified the $1.6 \mu \mathrm{m} 3 \rightarrow 6$ and the $2.29 \mu \mathrm{m} 0 \rightarrow 2$ band-head $\mathrm{CO}$ feature in a central core of FWHM of size around $45 \mathrm{pc}\left(0{ }^{\prime} 66\right)$. However, Thatte et al. (1997) estimate the contribution from stellar emission to be only $1 / 3$ of the total within an aperture of $1^{\prime \prime}$ radius. We note that the angular resolution they reached is only 0.9 , thus far below the SPHERE performance of $0 . ' 06$ in the $K_{s}$ band (see Sect. 2).

For the $K_{s}$-band profile, the contribution of hot dust from a compact central source was clearly identified as early as 1987 (Chelli et al. 1987). Thatte et al. (1997), based on their 0.' 4 resolution speckle observations estimated that essentially all the flux at $K_{s}$-band could be attributed to the point-like source. However, this is not what we observe on the $K_{s}$-band profile in Fig. 1, where the aisles extend far beyond those of the PSF. On the other hand, the wings beyond $17 \mathrm{pc}(0.24)$ are also very well fitted by a power law, which leads to the same reasoning as for the $H$ band, i.e. that it could correspond to stellar emission. If this hypothesis is correct, then it raises the question of why the exponents are so different between $K_{S}$ band and $H$ band.

Another question raised by this profile behaviour is how the cusp characteristics compare with those of cusps actually found in early-type galaxies or in LIRGs/ULIRGs. As regards the size of the cusp, by integrating the flux in discs of increasing diameter, we have determined a characteristic half-light radius of $99 \mathrm{pc}\left(1.420^{\prime \prime}\right)$ and $17 \mathrm{pc}\left(0.245^{\prime \prime}\right)$ for the $H$-band and $K_{s}$-band cusps, respectively, thus two very different scales that need to be explained.

Compared to the structures observed at millimetre wavelengths, either in molecular lines $(\mathrm{CO}, \mathrm{HCN})$ or in the continuum (García-Burillo et al. 2014, 2016; Imanishi et al. 2018), we note that the extent of the cusp at $H$ band reaches the inner radius of the off-centred circumnuclear molecular disc. This means that we do not expect significant extra extinction from the disc itself since the stellar cusp is almost entirely within its inner contour.

\subsection{Emission lines from the ISM}

We consider the possible role of differential dust extinction to explain the log-log $H$-band versus $K_{s}$-band slope difference in Sect. 3.3, but first let us examine another potential explanation: an extra contribution to the $K_{s}$-band flux by lines emitted by the ISM that would be significant and would feature a gradient. We can think of two kinds of line emission, either the emission of the (1-0) $\mathrm{S}(1)$ rotational-vibrational line $(2.1218 \mu \mathrm{m})$ of some excited $H 2$, or the $\mathrm{Br}_{\gamma}$ line $(2.1655 \mu \mathrm{m})$ associated with the narrow- or even the broad-line region. The first case must be excluded since the high angular resolution map of the line, obtained by Müller Sánchez et al. (2009) with the spectroimager SINFONI-VLT, clearly shows that the line emission is confined to a compact region (about $0.15^{\prime \prime}$ diameter) at $0.15^{\prime \prime}$ to the south-east of the central engine. Moreover, the same authors provided a spectrum of the region $0.3^{\prime \prime} \times 0.1^{\prime \prime}$ centred on the nucleus that clearly shows that the contribution of the (1-0) $S(1)$ line to the flux in the whole $K_{s}$ band is only $5 \times 10^{-4}$. The same spectrum indicates that the $\mathrm{Br}_{\gamma}$ line contribution to the same band is approximately $3 \times 10^{-3}$ and thus should also be excluded as an explanation for the differences in profile slopes between $H$ band and $K_{s}$ band. The same conclusion can be reached considering the long slit spectrum $\left(0.3^{\prime \prime}\right.$ slit width) in band $K_{s}$ band obtained by Gratadour et al. (2003).

\subsection{Variety of stellar cusps}

In the literature, the notion of central cusp seems to refer to a variety of situations or contexts and we first try to make this variety more explicit.

We can identify a first category where the cusp is associated with some compact central stellar cluster. For instance, it corresponds to an extra light distribution compared to a Sérsic distribution extrapolated to the very centre in early-type galaxies (Kormendy et al. 2009; Lauer et al. 2007). The expression nuclear cluster (NC) may also be used, either for early- or latetype galaxies (Böker et al. 2004). Lauer et al. (2007) use the notion of power-law galaxies (with steep brightness increase) as opposed to core galaxies where the central brightness is nearly constant. Côté et al. (2006) use the denomination compact central nucleus. We consider in the following that all these denominations represent the same kind of object, namely a central compact stellar cluster analogous to a globular cluster, even if it differs from this class by a higher luminosity (see e.g. Böker et al. 2004), typically 3.5 mag brighter. As stated by Böker (2008), NCs are common: the fraction of galaxies with an unambiguous NC detection is $75 \%$ in late-type (Scd-Sm) spirals (Böker et al. 2002), 58\% in earlier type (S0-Sbc) spirals (Balcells et al. 2007), and 70\% in spheroidal (E and S0) galaxies (Graham \& Guzmán 2003; Côté et al. 2006). The median characteristics that can be for instance derived from Table 2 of Lauer et al. (2007) is a half-light radius of $7 \mathrm{pc}$, within a range of 2-44 pc, and an exponent of 0.62 , within a range between 0 and 1.1. Côté et al. (2006) find for Virgo early-type galaxies a similar and consistent radius of $4.2 \mathrm{pc}$ for a range extending to $62 \mathrm{pc}$. These typical characteristics do not depend strongly on the type of galaxy; for instance, Böker et al. (2004) find, for a sample of late-type galaxies, a histogram of radii with a first peak at $3 \mathrm{pc}$ and another one at $8 \mathrm{pc}$, the range extending to $30 \mathrm{pc}$. However, it has been clearly established that faint ellipticals $\left(M_{V} \leq 20\right)$ exhibit a more cuspy profile than massive ones (Kormendy et al. 2009; Côté et al. 2006), a feature that likely translates into a different history of merging.

The second and distinct category of stellar cusps we have identified in the literature is more clearly related to recent or ongoing starbursts. We refer in particular to the study by Haan et al. (2013) of a large sample of LIRGs and ULIRGs where HST images in the NIR were used to derive the characteristics of the nuclear stellar cusps they found in nearly all objects. We analysed the data compiled by those authors concerning $\approx 80 \mathrm{LIRG}$ and ULIRG objects, a large fraction of which are mergers at various phases of the process. In most of the objects the authors found a cuspy profile in the nuclear region. They provide a table of the various properties of the selected objects, including radius and NIR luminosity of the cusp, as well as the exponent of the cuspy brightness distribution. Clearly the average characteristics are distinct from those of the class we discussed above: we find a median half-light radius of $475 \mathrm{pc}$ within a range between $46 \mathrm{pc}$ and $2.4 \mathrm{kpc}$, while the exponent of the cusp has a median value of 0.80 within a range between 0.1 and 1.7. The cusp radius is remarkably much larger than for the nuclear cluster of the class identified above, and the exponent is significantly larger and can reach high values.

Finally, the third category of cusps that is described in the literature corresponds to the compact stellar population that is distributed around a massive black hole in the centre of a galaxy. Some theoretical works have explored the dynamics of this peculiar system (see e.g. Preto 2010 for a review), but there are no well-established observational facts, with the notable exception of the centre of the Milky Way 
(Genzel et al. 2003; Schodel et al. 2017). These nuclear stellar cluster (NSC) cusps are characterised by half-mass or half-light radii of $1-5 \mathrm{pc}$ and are in principle the result of dynamical effect, namely two-body relaxation through frequent encounters of stars or stellar $\mathrm{BH}$ in the strong gravitational field of a massive BH. In particular, Bahcall \& Wolf (1976) established the first solid analytical expression of the stellar distribution around a massive $\mathrm{BH}$ and showed that it must be cuspy with an exponent $1.75(7 / 4)$. Refinements to the theory were brought later by numerous teams (for a review, see e.g. Preto 2010), especially the fact that there must be a mass segregation with different exponents according to the mass of the stellar objects, the most massive stellar BH having the largest exponent (1.8) and main sequence stars a lower value (typically 1.3) (Freitag et al. 2006). These exponents are thus clearly larger than that found in the two previous classes we examined, and closer to the ones observed in NGC 1068, but if we consider both the predicted small half-mass radius and the too large relaxation time $T_{\mathrm{r}}$ required to reach a cuspy distribution due to the large mass of the $\mathrm{BH}$ - we can no longer consider this case for NGC 1068.

To make the distinction between the two classes more readable in the following, we will call the first the nuclear cluster cusp (NCC) class, and the second the central starburst cusp (CSC) class. Our goal is to try to determine if there are clues that allow the identification of the cusp we observe with one of these classes, or a combination of the two.

The rather large exponent we found for the cusp in NGC 1068 and the rather wide half-light radius clearly rule out the case where we would observe a mere NCC; such large exponents are never observed for this class.

For the CSC class, NGC 1068 shares, at least for the $H$-band cusp, some characteristics, with an exponent and a radius that are indeed significantly different from the median values, but within the observed ranges for this class. Nevertheless, the $K_{S^{-}}$ band profile with its small half-light radius and large exponent could weaken this statement, but we show in Sect. 4 that the dust emission cannot be neglected. However, there is such a large dispersion of characteristics in the sample of Haan et al. (2013) that the question deserves closer examination.

To go one step further, starting from the data set compiled by Haan et al. (2013), we tried to determine whether some general law could relate the parameters of the cusp in CSC. The purpose was to see if NGC 1068 could obey such a general law even if it is not considered a LIRG. If the answer were yes, it would be a strong indication that the cusp is the result of a starburst evolution rather than the result of a dynamical effect around a massive BH. We first selected the 62 objects where the brightness distribution is indeed cuspy $(\gamma \neq 0)$ and where the information on the three parameters: radius, NIR luminosity, and exponent of the cusp, was complete (e.g. not given as an upper or lower limit). We looked for a fit of $\gamma$ as a linear combination of the log of the NIR cusp luminosity $L_{\text {cusp }}$ and the log of the cusp radius $R_{\text {cusp. }}$. We found that the law

$\gamma=1.133 \times\left[\log 10\left(L_{\text {cusp }} / R_{\text {cusp }}\right)-6.63\right]$

relating the three parameters was rather robust, with a covariance between the actual value and the predicted value of 0.85 . The robustness of the fit can be seen in Fig. 5, where the luminosity of each selected object is plotted against the value predicted by the law using the two other parameters.

The straightforward interpretation of the law is that at a given luminosity the smaller the radius of the cusp, the steeper the distribution and, conversely, a lower luminosity corresponds to a

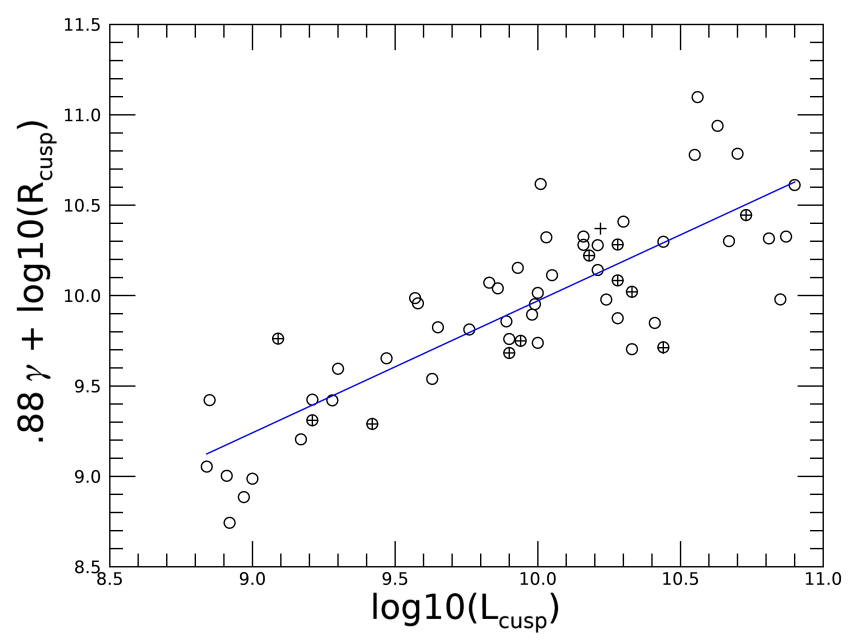

Fig. 5. Estimated cusp luminosity of a selection of the objects studied by Haan et al. (2013), as predicted by the phenomenological law we found (see text) vs the actual cusp luminosity. The blue line is the linear regression. The cross indicates the objects that are not mergers or are in a wide system.

smoother cusp. We note that in Fig. 5, we have flagged with an additional cross the objects that do not belong to a merger or that are members of a system with widely separated galaxies (class 0 and 1 in Haan et al. 2013). We cannot see any trend indicating that those objects do not follow the general law we found. This is important since, as noted by Haan et al. (2013), the fact that there is no obvious merging situation does not prevent a cusp from being present.

In order to compare the characteristics of the NGC 1068 cusp with the derived law, we have to estimate the NIR luminosity of the cusp. Because the obscuration is extremely high in the central region, we cannot consider visible data as was done in the previously mentioned studies of cusps in galaxies.

To be consistent, we consider only the $H$-band data which are less affected by possible dust emission, assuming that it represents well the stellar population distribution. With a calibration done with the star BD-00413, observed the same night, we evaluate a magnitude $m_{H}=9.5$ within an aperture of radius $100 \mathrm{pc}$. Assuming a stellar population with $H-K_{s}$-band $\approx 0$ and an age between $4.0 \times 10^{8}$ and $6.0 \times 10^{9} \mathrm{yr}$, the bolometric luminosity would be between $3.7 \times 10^{9}$ and $1.8 \times 10^{10} L_{\odot}$, according to Fig. 5 in Thatte et al. (1997). This is consistent with Davies et al. (2007) who derives a bolometric luminosity of stars in the centre of $7 \times 10^{9} L_{\odot}$. Using these estimates and the half-light radius $100 \mathrm{pc}$ in Eq. (1), we find a predicted exponent between 1.06 and 1.84 , a range that brackets the exponent 1.3 observed on the $H$-band brightness profile.

This being said, we must emphasise the somewhat large uncertainty linked to the evaluation of the bolometric stellar luminosity. This rather large exponent of 1.3 is also partly consistent with the value presented by Müller Sánchez et al. (2009), who estimated an enclosed stellar mass varying linearly with the radius $\left(M_{\star}(r) \propto r\right)$, thus requiring a stellar density varying as $r^{-2}$. The agreement with the empirical law we established suggests that the cusp in NGC 1068 could be closely related to some starburst activity. For Davies et al. (2007) there are indeed strong indications, based on $\operatorname{Br} \gamma$ equivalent width, supernova rate, and mass-to-light ratio, that the stellar nucleus of NGC 1068 is the remnant of recent but no-longer-active starburst episodes. The authors estimate the age of the last episode to be 200-300 Myr. 


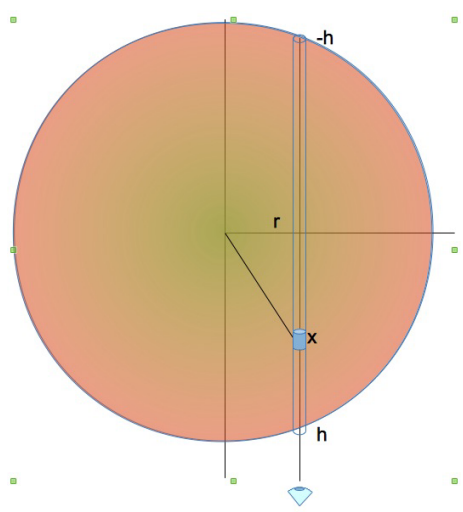

Fig. 6. Sketch of the simple radiative transfer model we built in order to simulate the differential $\mathrm{H}-/ K_{s}$-band extinction effect of a cuspy distribution of dust in which a cuspy distribution of stars is embedded.

The only viable class to which the NGC 1068 cusp appears to be related is the CSC. We note that Mushotzky et al. (2014), using Herschel data on a sample of X-ray selected AGNs, reach the conclusion that most AGN live in high star formation nuclear cusps. However one question remains: if we see mainly stellar emission, why are the exponents and half-light radii so different at $H$-band and $K_{s}$-band? A first tentative explanation is that this difference is due to the effect of extinction by dust which may play an important role in this region. Another possibility is that there is a segregation in stellar population, with redder giant stars dominating towards the most central part. A combination of the two effects is also possible. We examine these two cases in the next subsections.

\subsection{Pure differential dust extinction}

The larger dust absorption at $H$ band compared to $K_{s}$ band must smooth the stellar brightness more strongly as we approach the central peak, provided that the dust quantity increases inward. To test this effect, we developed a rather simple radiative transfer model, assuming that the ISM in which the cuspy distribution of stars is embedded also follows a cuspy, i.e. power-law, distribution, but with possibly a different exponent. Figure 6 depicts the geometry used.

At a given wavelength, the brightness at distance $r$ from the central peak is given by

$\left.F(r) \propto \int_{-h}^{h} L_{\star}(\lambda) \times n_{\star}\left(\sqrt{(} r^{2}+x^{2}\right)\right) \times \exp (-\tau(x)) \mathrm{d} x$,

where $n_{\star}(r)$ is the radial density of star, $L_{\star}(\lambda)$ the star luminosity at $\lambda$, and $\tau(x)$ the optical depth between the observer and the cell at location $x$ on the line of sight:

$\left.\tau(x)=\int_{-h}^{x} \alpha_{\text {dust }}\left(\sqrt{(} r^{2}+x^{2}\right)\right) \mathrm{d} x$,

with $\alpha_{\text {dust }}(r)$ the extinction coefficient of dust per unit of length at radius $r$. Indeed, $\alpha_{\text {dust }}$ depends on the wavelength, being proportional to $Q_{\text {ext }}(\lambda)$.

We developed a numerical code in order to look for the exponents of the cuspy distribution of dust and of the star density that would give the best fit to the observed $H$-band and $K_{s}$-band radial distributions. Using the Powell minimisation algorithm and leaving free both exponents, we minimised the square of the $\log$ of the ratio of the observed to simulated profiles at $H$-band and $K_{s}$-band simultaneously. We set $\tau_{K_{s}} / \tau_{H}=0.61$,

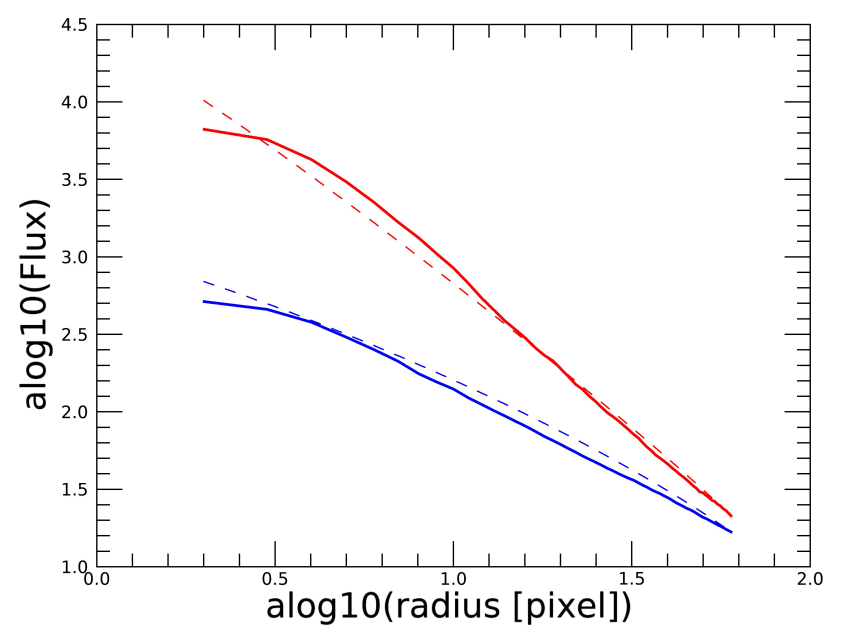

Fig. 7. Red and blue solid lines: observed 2 to $50 \mathrm{pc}$ radial fluxes in $K_{s}$ and $H$ band; red and blue dashed lines: $K_{s}$-band and $H$-band profiles resulting from our radiative transfer model, when the power law for the stellar and dust radial density have exponents of -4.20 and -1.05 , respectively. The result is a quasi-power law for both brightness profiles.

i.e. the value found in the standard ISM. We reached a solution which is rather satisfactory, as shown in Fig. 7 where the radial fluxes at $K_{s}$ and $H$ band are plotted, modelled using a power law for the stellar radial density of exponent -4.2 and a dust density varying as $r^{-1.05}$. In Fig. 8 we plot the deduced radial distribution of the optical depth at $H$ and $K_{s}$ band down to the centre of the cusp: it appears cuspy as well. From this plot we can derive a radial optical depth $\tau_{H}=7.7$ and $\tau_{K_{s}}=4.7$ between $2.5 \mathrm{pc}$ and $60 \mathrm{pc}$; this corresponds to $\tau_{V}=43$ in $V$.

This value would be close to twice that derived by Burtscher et al. $(2015,2016)$ who estimate $A_{v}=24.6$. If our model is correct, the need for some extra extinction arising in the so-called torus would not be required, and the dusty cusp would even have to be truncated at a radius of several pc; in other words, the torus could be identified with the tip of the dusty cusp. On the other hand, Grosset et al. (2018) used their Monte Carlo radiative transfer code Montagn (Grosset et al. 2016) to interpret AOassisted NIR polarisation measurements (Gratadour et al. 2015) and to estimate a minimum optical depth of 20 at $K_{s}$ band to the CE (see also next section). In this case, the dusty cusp we suppose here would not be sufficient to explain the most central extinction, and a torus-like extra structure is still needed. Nevertheless, it is also possible that dichroism is also involved in the polarisation seen at the centre, in which case a lower extinction would be required (Grosset et al., in prep.).

If indeed the exponent we derive for the dust and stellar cusps are real, they need to be explained on some theoretical basis. In particular, the exponent of -4 for the stellar density appears extremely steep, and is predicted in none of the theoretical studies we are aware of. We explore in the next subsection the possible role of a segregation of the stellar population with radius, where the intrinsic $H-K_{s}$ colour would increase when approaching the centre.

\subsection{Combination of differential dust extinction and stellar type segregation}

We introduced in our numerical model the new constraint that the quantity $H-K_{s}$ varies as the Log of the radius: 


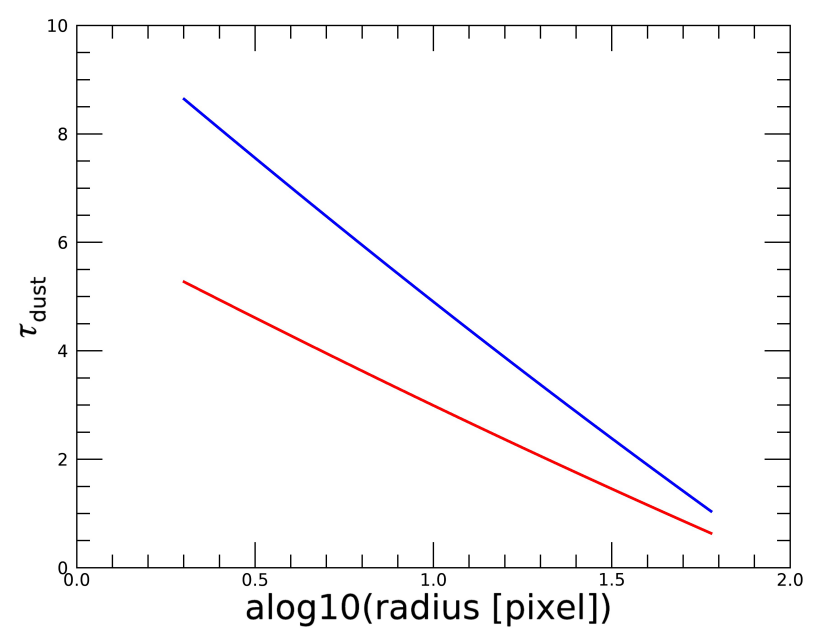

Fig. 8. Radial distribution of the optical depth in $H$ band (blue) and $K_{s}$ band (red) in the model providing the best fit to the $H$-band and $K_{s}$-band observed brightness profiles (see text).

$H-K_{s}=C \times \log 10\left(r / r_{\max }\right) / \log 10\left(r_{\text {in }} / r_{\text {max }}\right)$, assuming thus that $H-K_{s}=0$ at $r_{\max }$ and $H-K_{s}=C$ at $r_{\text {in }}$. This means that the un-reddened ratio Flux $_{K_{s}} /$ Flux $_{H}$ varies as a power law versus $r$ as $r^{C / 2.5 / \log 10\left(r_{\text {in }} / r_{\max }\right)}$. For instance, if $r_{\text {in }}=1 \mathrm{pc}$ and $r_{\max }=100 \mathrm{pc}$, the exponent of the power law would be $-C / 5$ On the other hand, the maximum value of $C$, assuming that the very central region is dominated by red giants is 0.3 (e.g. Ducati et al. 2001). We cannot thus expect a power law much steeper than $r^{-.06}$, and the effect on the final fit, after the introduction of this new ingredient in the model, cannot be to change significantly the exponent of the stellar cusp nor of the dusty cusp.

This was indeed confirmed by performing the fit that leads to the values of exponents -3.75 and -1.27 for respectively the stellar and the dusty cusps. The stellar cusp exponent is now somewhat less extreme than the value -4.05 we previously determined, but it still remains very large compared to any prediction made by numerical simulations or even observed in the well-documented cusp of the Galactic Centre (Genzel et al. 2003; Schodel et al. 2017) where the exponent is -2.0 for $0.4 \mathrm{pc}$ $<r<4$ pc. We note, however, that the mass of the black hole SgrA* is significantly smaller than in NGC 1068.

\subsection{Colours and luminosity of the stellar cusp}

So far our aim has been essentially to reproduce the $H$-band and $K_{s}$-band slopes of the radial profiles without considering the measured fluxes themselves. Let us now look to see if there is some consistency between the estimated fluxes of the stellar cusp and the model we developed. The measured $H$-band and $K_{S}$-band magnitudes of the cusp alone, within a $1^{\prime \prime}$. 47 diameter aperture $(102 \mathrm{pc})$ centred on the peak, are respectively 9.51 and 8.63. Assuming that we correctly evaluated the central pointsource magnitude in $K_{s}$ band (see Sect. 5), we derive then for the supposed stellar cusp a magnitude $K s_{\text {cusp }}=8.9$. This means an observed colour $\left(H-K_{s}\right)_{\text {cusp }}=0.6$. Now, if we compute in our model, for each filter, the ratio of the intrinsic cusp luminosity to the one escaping from the dusty cusp, we can derive the corresponding global $\left(H-K_{s}\right)$ due to reddening. It amounts to 1.6 , assuming the second version of our model, i.e. combining differential dust extinction and stellar type segregation. The intrinsic $\left(H-K_{S}\right)_{\text {cusp }}^{0}$ would then be -1.0 . Even considering the uncertainties and the crudeness of the model, this value appears inconsistent with a stellar population. For instance, for a B1 star $\left(H-K_{s}\right)=-0.14$ (Koornneef 1983) and a very young stellar cluster $\left(H-K_{s}\right)=-0.1-0.2$ (Santos et al. 2013). This is a first clue that the hypothesis of pure stellar cusp is not robust enough.

Now the apparent luminosity of the cusp at $K_{s}$ band amounts to $L_{K s}=3.210^{8} L_{\odot}$. On the other hand, our model gives a fraction of escaping stellar light at $K_{s}$ band of $5.5 \%$, leading to an intrinsic $K_{s}$-band luminosity of $4.8 \times 10^{9} L_{\odot}$. This value is clearly too high since the ratio $L_{\text {bol }}$ to $L_{K s}$ is at least 20 for a cluster of 5 Gyr (e.g. see Fig. 5 in Thatte et al. 1997), leading to a total intrinsic stellar luminosity of the cusp of $>1 \times 10^{11} L_{\odot}$, i.e. between 1/4 (Raban et al. 2009) and 2/3 (Bock et al. 2000) of the nuclear bolometric luminosity. This is probably the point that invalidates the interpretation in terms of pure stellar cusp: this fraction is much larger than usually accepted, especially if we consider that a cluster $5 \mathrm{Gyr}$ old of this luminosity would have a mass of $2.8 \times 10^{11} M_{\odot}$ according to Thatte et al. (1997; Fig. 5). In addition, the involved quantity of dust and thus of gas would appear unrealistic as well.

\section{Warm/hot dust extended emission}

We examine now whether there is an alternative interpretation of the power-law behaviour of at least the $K_{s}$-band brightness radial profile in terms of thermal emission by warm to hot dust heated by the energetic radiation from the central engine. Of course this interpretation needs to be somewhat consistent with several known properties of the nuclear region.

One first requirement is that the region where the thermal radiation dominates over other sources, especially stellar radiation, must extend at least to $50-60 \mathrm{pc}$, i.e. where the cuspy distribution is clearly observed. This requirement means that the dust temperature cannot drop rapidly along a radius, or in other words, that the heating radiation from the CE cannot be attenuated on a small scale-length. A very rough estimate of what this requirement means can be made as follows. Let us consider that the power law in $r^{-2}$ is verified in the range $6-60 \mathrm{pc}$, thus that the ratio of thermal emission integrated on the line of sight between 6 and $60 \mathrm{pc}$ should be $1 / 100$. Assuming that the medium is optically thin and homogeneous, the dust luminosity per unit volume between 6 and $60 \mathrm{pc}$ must be in the ratio $1 / 1000$ because the effective path on which significant emission is integrated towards the observer is 10 times longer at $60 \mathrm{pc}$. Assuming a radius of sublimation $R_{\text {sub }}=2 \mathrm{pc}$, a temperature of dust sublimation $T_{\text {sub }}=1500 \mathrm{~K}$, and a dust coefficient of absorption $Q_{\mathrm{abs}}(\lambda) \propto \lambda^{-2}$, the dust temperature would vary as $T(r)=1500\left(r / R_{\text {sub }}\right)^{-2 / 6}$ if the heating flux varies as $r^{-2}$, i.e. if it is not attenuated. In that case, the ratio of dust luminosity per unit volume between 6 and $60 \mathrm{pc}$ would then be approximatively 1000 at $2.2 \mu \mathrm{m}$, as required. The hypothesis that the CE light is not attenuated may seem an unrealistic constraint because we know that, at least on the direct line of sight to the CE, there must be an extinction $A_{v} \approx 20$ attributed to the torus. However, this does not prevent, in the dust-free bicone perpendicular to the torus, the extinction from being low enough, so that a significant fraction of the volume around the CE can be illuminated directly by the energetic photons. An alternative case could be that the medium is sufficiently clumpy so that a fraction of direct light can indeed reach regions distant from the $\mathrm{CE}$ and heat the local dust at a high enough temperature. Scattering on the surface of clumps should also help energetic photons to travel those rather large distances. This is, in fact, the situation that we will favour in the following. To assess how realistic this hypothesis is, we built a model of a population of spherical absorbing/scattering 


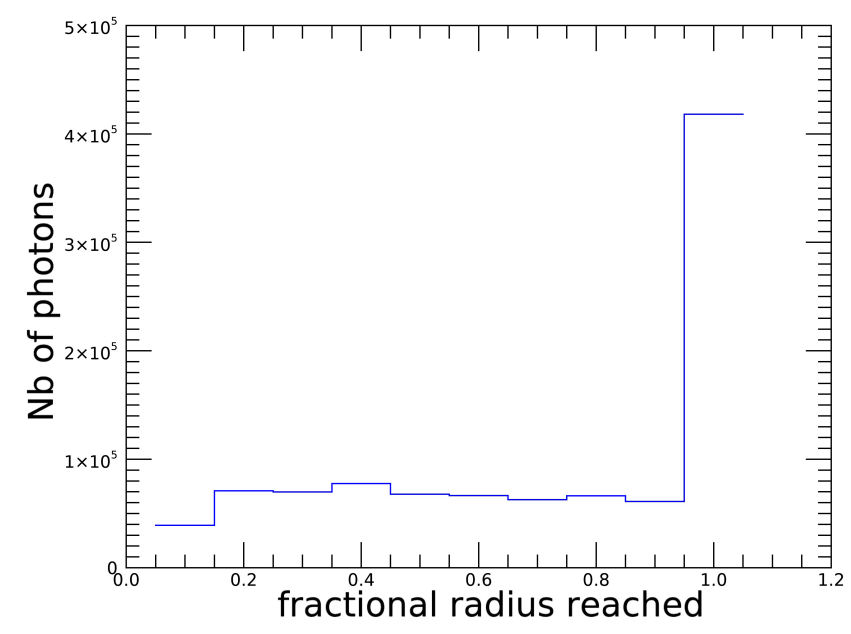

Fig. 9. Histogram of the radius reached by photons launched from the centre of a sphere populated by 300 absorbing clouds totalling a filling factor of $2.5 \%$. The last and highest bin corresponds to escaping photons. There is a very flat distribution of radii.

clouds of various radii (uniformly distributed between 0 and $1.6 \mathrm{pc}$ ) within a sphere of radius $50 \mathrm{pc}$ and launched $10^{6}$ photons. For a volume filling factor of $2.5 \%$ and 300 clouds, the probability for a photon to reach the external radius of the sphere without being absorbed is $42 \%$. Figure 9 illustrates the histogram of radii reached by photons in such a simulation, where an albedo of 0.5 was taken. We note the very flat distribution of radii.

Assuming thus that dust can be heated significantly at least $50 \mathrm{pc}$ away from the $\mathrm{CE}$, we adapted the numerical code developed for the stellar cusp simulation (see Sect. 3.3) to model the situation described above. The luminosity of the dust per unit volume is simply taken proportional to Planck's law at the local temperature. We also assume that there could be some extinction on the line of sight. We note that this last ingredient is not in contradiction with the idea that direct light from the $\mathrm{CE}$ can reach the dust at any distance because this last assumption stems from the clumpiness of the medium, while the extended dust thermal emission could suffer extinction by clumps along the line of sight.

By adjusting very few parameters, namely a sublimation radius of $1 \mathrm{pc}$, a constant dust density and $\tau_{K s}=6.5$ from the centre to $50 \mathrm{pc}$, we are able to fit fairly well the measured $K_{s}$-band radial profile, as illustrated in Fig. 10 by the red dashed line. However, despite the quality of the fit, the required $K_{s}$-band extinction is fairly large and hardly compatible with our hypothesis of heating by light directly from the CE. Moreover, when examining the case of the $H$ band, assuming that here too dust thermal emission is dominant, we find no solution by our model that is consistent with the parameters found for the $K_{s}$-band fit. The most acceptable solution indicated by the dotted blue line in Fig. 10, requires $\tau_{H}=14.5$ a value inconsistent with the classical grain extinction curve where $\tau_{K s} / \tau_{H}=0.61$ (against 0.44 here) and, moreover, no fit is found. In particular, the temperature at large distances is too low to produce a significant flux in $H$.

The inescapable conclusion is that we do not actually detect dust thermal emission in $H$ band, but only light of a stellar cusp. This leads us to consider finally, rather than the pure $K_{s}$-band profile, the same one from which the stellar cusp contribution is subtracted, assuming that the contribution is just proportional to the $H$-band profile. We used a weighting factor of 0.8 on the subtracted $H$-band profile. This corresponds to $H$ - $K_{s}=0.25$, a value usually found in globular clusters. We obtain the green profile in Fig. 11. A fairly good fit by our model is found (green dashed

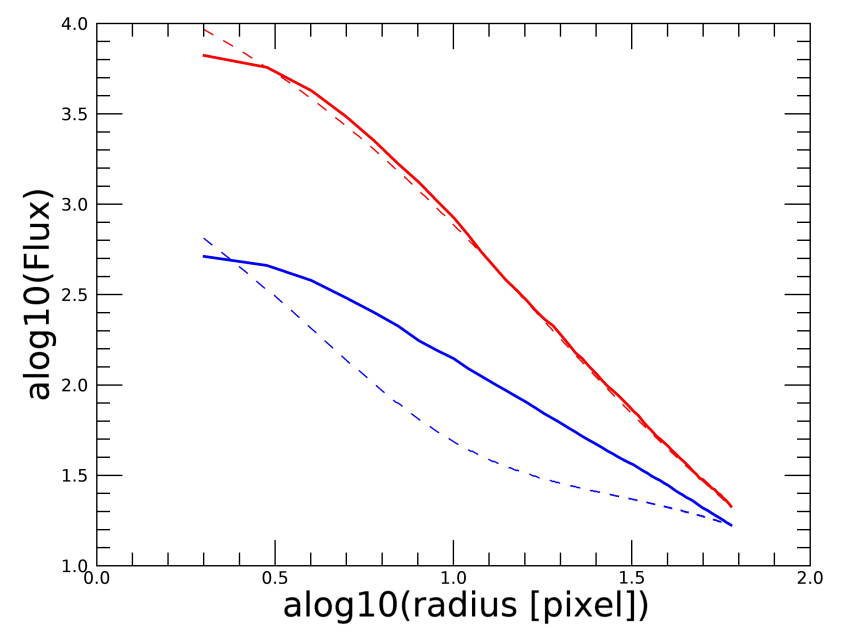

Fig. 10. $K_{s}$-band (red solid) and $H$-band (blue solid) radial profiles. Red dashed line: fit to the $K_{s}$-band profile assuming a maximum $\tau_{K}=6.5$ on the line of sight. Blue dashed line: $H$-band profile of dust emission with $\tau_{H}=15$ (see text).

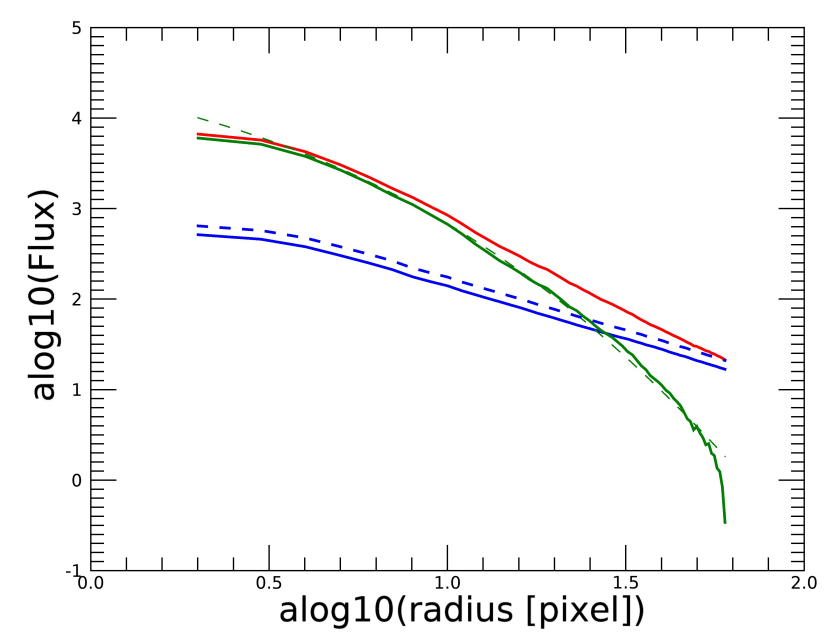

Fig. 11. $K_{s}$-band (red solid) and $H$-band (blue solid) radial profiles; green solid line: $K_{s}$-band radial profile subtracted with a stellar cusp contribution proportional to the $H$-band profile; blue dashed line: estimated stellar cusp at $K_{s}$; green dashed line: best fit by the model of thermal dust emission ( see text).

line in Fig. 11) now with $\tau_{K_{s}}<1.5$, a range quite consistent with the hypothesis of clumpiness. We actually assume $\tau_{K_{s}} \ll 1$ in the following.

Given the assumed low extinction, the $K_{s}$-band magnitude of the hot dust can be estimated at 9.55. The spectral luminosity at $K_{s}$ band is then $5.0 \times 10^{34} \mathrm{~W}$, which can be translated into $L_{\text {bol }}=3.3 \times 10^{37} \mathrm{~W}$, using our model. With the same model, we can relate this luminosity, derived from observation, to the content in warm dust, using some classical assumptions on the dust: MRN (Mathis Rumpl Nordsiek) size distribution as $a^{-3.5}$, $a_{\min }=0.5 \mathrm{~nm}, a_{\max }=500 \mathrm{~nm}, Q_{\mathrm{abs}}$ from Draine (1985). The mass of dust accounting for the observed luminosity, using the temperature distribution of our model, is $M_{\text {dust }}=0.07 M_{\odot}$, a value which definitely corresponds to a very diluted medium. For instance, we derive a typical hydrogen density of about $2000 \mathrm{~m}^{-3}$, so that assuming $\tau_{K s} \ll 1$ is thus fully justified.

We think that with this final configuration we have reached a fully consistent picture where the nature of the two cuspy distributions in $H$ band and $K_{s}$ band are in fact largely different: 
it is totally dominated by a stellar cusp at $H$ band and, at $K_{s}$ band, it is progressively dominated by warm to hot dust thermal radiation when going inward, this hot dust belonging to a very diluted medium. We now can understand why the half-light radii at the $H$-band and $K_{s}$-band cusps, correspond to very different scales, as noted previously: the dust thermal emission dominating the central region in $K_{s}$ is indeed much more compact than the stellar cusp seen in $H$.

\section{Nature of the central point-like source}

As shown in Sect. 2, the $K_{s}$-band radial profile reveals that there is a quasi-point-like source superimposed on the power-law aisles discussed above. Using the SPHERE calibration provided by ESO and estimating the best PSF fitting to the point source, we find $\mathrm{m} K s_{\text {point }}=10.2 \pm 0.2$. The point-source provides $31 \%$ of the $K_{s}$-band luminosity measured in the $50 \mathrm{pc}$ diameter central part. This number is significantly smaller than that estimated by Thatte et al. (1997) from their speckle and spectroscopic data, albeit at a lower spatial resolution.

We estimated that the size of the source cannot be larger that 1.7 pc. As proposed by several authors (Thatte et al. 1997; Gratadour et al. 2003), a straightforward interpretation is that the source corresponds to the emission by dust at high temperature, close to sublimation, at the internal walls of the torus. We considered various estimates of this internal radius. Nenkova et al. (2002) propose the relation $R_{\text {in }}=1.2 L_{12}^{1 / 2} \mathrm{pc}$, with $L_{12}=10^{-12} L_{\mathrm{bol}} / L_{\odot}$, which leads to $R_{\mathrm{in}}=0.9 \mathrm{pc}$, using $L_{\mathrm{bol}}=2.1 \times$ $10^{45} \mathrm{erg} \mathrm{s}^{-1}$ (Marconi et al. 2004); with $L_{\text {bol }}=4.2 \times 10^{44} \mathrm{erg} \mathrm{s}^{-1}$ as estimated by García-Burillo et al. (2014) we instead find $R_{\text {in }}=$ $0.4 \mathrm{pc}$. This is to be compared to the maximum radius of $0.8 \mathrm{pc}$ we mentioned in Sect. 2 for the central point source. There is thus a good consistency with the picture suggested by our SPHERE data.

As an additional step, we can compare the flux we measure for the point source to an estimate of the power emitted by the assumed hot dust. At the distance of NGC 1068, $K_{s}$ band $=10.2$ means a $K_{s}$-band spectral luminosity of $2.9 \times 10^{34} \mathrm{~W} \mu \mathrm{m}^{-1}$. Assuming a sublimation temperature of $1400 \mathrm{~K}$ and a central cavity radius of $0.6 \mathrm{pc}$ with walls optically thick at $2.2 \mu \mathrm{m}$, the expected intrinsic $K_{s}$-band spectral luminosity from the cavity would be

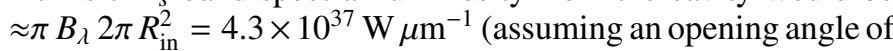
the bicone of $120^{\circ}$ ). This means that a $7.9 \mathrm{mag}$ of extinction in $K_{s}$ band is required to reconcile the two numbers. This is significantly lower than the 20 mag in $K_{s}$ band that Grosset et al. (2018) have proposed to explain their NIR polarisation data, but somewhat higher than the range $2.5-4.8$ of extinction proposed by several authors (Thatte et al. 1997; Efstathiou et al. 1995; Young et al. 1995). Given our rough estimate of the $K_{s}$-band luminosity of the very host dust, which is based on an approximate $R_{\text {in }}$ and geometry, neglects any radiative transfer effect, including scattering, and does not consider a realistic dust composition, the uncertainty is probably larger than $2.5 \mathrm{mag}$. In any case, the essential of the obscuration must occur in the very central part, thus supporting the hypothesis of a torus with a dense internal shell at a pc scale. This does not prevent a more extended molecular extension of the torus from existing on a $7-10 \mathrm{pc}$ scale, like the one identified by García-Burillo et al. (2016), and even a more diluted envelope on a $30 \mathrm{pc}$ scale, as proposed by Gratadour et al. (2015) in order to explain the peculiar NIR polarisation pattern.

\section{Conclusion and prospectives}

The high angular resolution NIR images of the central $200 \mathrm{pc}$ of of NGC 1068 reveal a cuspy distribution of the brightness at all wavelengths between $H$ band and $K_{s}$ band with, however, a continuously increasing absolute value of the exponent with wavelength. Using a simple numerical model, we tried first to interpret this behaviour as resulting from a very cuspy $\left(r^{-4}\right)$ distribution of the stellar density in a central compact cluster down to $1 \mathrm{pc}$, while the $H-/ K_{s}$-band cusp exponent difference would be accounted for by a distribution of the ISM, also cuspy but with a less steep exponent $\approx-1$. Introducing some segregation in the stellar population, i.e. assuming that giant stars are dominating towards the very centre, only slightly changes the exponent of the stellar cusp that becomes -3.75 , i.e. a value never observed nor predicted on theoretical grounds. The required dust opacity is in all cases very high, so that when computing the intrinsic luminosity of the putative stellar cluster, we reach a value much too high to be realistic.

The alternative interpretation that we explored is that the $K_{s}$-band brightness profile is a combination of a stellar cusp and warm to hot dust emission and it appears clearly more acceptable. Considering the residual profile when a stellar cusp component, derived from the $H$-band profile, is subtracted, a good fit can be obtained by a simple model of dust heated by the CE in a homogeneous and diluted medium.

In addition to the cusp and thermal warm dust radiation, the $K_{s}$-band profile features a distinct central point-like source interpreted as the very hot dust close to the central engine. The estimated extinction to the very centre is consistent with previous estimates. The overall picture revealed by this study appears closely consistent with the scheme of a clumpy medium around the $\mathrm{CE}$ and with the requirement of a torus on a pc scale. It also brings constraints on the structure of the ISM within the 100 central pc, which must be very diluted.

Finally, we note that the three components we identified within the central $100 \mathrm{pc}$, namely the stellar cusp, the very hot dust core, and the extended warm dust, each contribute to the $K_{s}$-band luminosity for a similar fraction, with magnitudes in $K_{s}$-band respectively of 9.8, 10.2, and 9.6.

Several questions are opened by this study, and here we mention a few of them. Can a similar stellar cusp and extended dust emission be observable in other AGNs thanks to HAR imaging or even interferometry? Can radiative transfer models produce a consistent view of all observations, including recent ALMA molecular detection of the torus, mid-IR imaging, mid-IR interferometry, and NIR polarisation? Can the rather steep stellar cusp be predicted by theoretical models combining multi-scale hydrodynamics, stellar dynamics, and star formation? Is the relationship that we established from the data of Haan et al. (2013) between cusp radius, cusp luminosity, and exponent verified for other cases of nearby AGNs? Is there a solid explanation for this phenomenological law, especially that the exponent depends only on the ratio $L_{\text {cusp }} / R_{\text {cusp }}$ ? In any case, it is in the views of our team to tackle the two first questions, especially the second by using our numerical model Montagn.

Acknowledgements. The authors would like to acknowledge financial support from the Programme National Hautes Energies (PNHE) and from "Programme National de Cosmologie and Galaxies" (PNCG) funded by CNRS/INSU-IN2P3INP, CEA, and CNES, France.

\section{References}

Antonucci, R. R. J., \& Miller, J. S. 1985, ApJ, 297, 621

Bahcall, J. N., \& Wolf, R. A. 1976, ApJ, 209, 214

Balcells, M., Graham, A. W., \& Peletier, R. F. 2007, ApJ, 665, 1084

Bian, W.-H., \& Zhao, Y.-H. 2003, PASJ, 55, 599

Bland-Hawthorn, J., Gallimore, J. F., Tacconi, L. J., et al. 1997, Ap\&SS, 248, 9 
Bock, J. J., Neugebauer, G., Matthews, K., et al. 2000, AJ, 120, 2904 Böker, T. 2008, ApJ, 672, L111

Böker, T., Laine, S., van der Marel, R. P., et al. 2002, AJ, 123, 1389

Böker, T., Walcher, C. J., Rix, H. W., et al. 2004, in The Formation and Evolution of Massive Young Star Clusters, eds. H. J. G. L. M. Lamers, L. J. Smith, \& A. Nota, ASP Conf. Ser., 322, 39

Burtscher, L., Orban de Xivry, G., Davies, R. I., et al. 2015, A\&A, 578, A47

Burtscher, L., Davies, R. I., Graciá-Carpio, J., et al. 2016, A\&A, 586, A28

Chelli, A., Perrier, C., Cruz-Gonzalez, I., \& Carrasco, L. 1987, A\&A, 177, 51

Côté, P., Piatek, S., Ferrarese, L., et al. 2006, ApJS, 165, 57

Davies, R. I., Müller Sánchez, F., Genzel, R., et al. 2007, ApJ, 671, 1388

Draine, B. T. 1985, ApJS, 57, 587

Ducati, J. R., Bevilacqua, C. M., Rembold, S. B., \& Ribeiro, D. 2001, ApJ, 558, 309

Efstathiou, A., Hough, J. H., \& Young, S. 1995, MNRAS, 277, 1134

Freitag, M., Amaro-Seoane, P., \& Kalogera, V. 2006, ApJ, 649, 91

García-Burillo, S., Combes, F., Usero, A., et al. 2014, A\&A, 567, A125

García-Burillo, S., Combes, F., Ramos Almeida, C., et al. 2016, ApJ, 823, L12

Genzel, R., Schödel, R., Ott, T., et al. 2003, ApJ, 594, 812

Goodman, J. 2003, MNRAS, 339, 937

Graham, A. W., \& Guzmán, R. 2003, AJ, 125, 2936

Gratadour, D., Clénet, Y., Rouan, D., Lai, O., \& Forveille, T. 2003, A\&A, 411, 335

Gratadour, D., Rouan, D., Mugnier, L. M., et al. 2006, A\&A, 446, 813

Gratadour, D., Rouan, D., Grosset, L., Boccaletti, A., \& Clénet, Y. 2015, A\&A, 581, L8

Grosset, L. 2017, Thesis, Université Paris Sciences et Lettres, France

Grosset, L., Marin, F., Gratadour, D., et al. 2016, in SF2A-2016: Proceedings of the Annual meeting of the French Society of Astronomy and Astrophysics, eds. C. Reylé, J. Richard, L. Cambrésy, et al., 57
Grosset, L., Rouan, D., Gratadour, D., et al. 2018, A\&A, 612, A69 Haan, S., Armus, L., Surace, J. A., et al. 2013, MNRAS, 434, 1264 Hopkins, P. F., \& Quataert, E. 2010, MNRAS, 407, 1529

Hopkins, P. F., \& Quataert, E. 2011, MNRAS, 415, 1027

Hopkins, P. F., Hayward, C. C., Narayanan, D., \& Hernquist, L. 2012, MNRAS, 420, 320

Imanishi, M., Nakanishi, K., Izumi, T., \& Wada, K. 2018, ApJ, 853, L25

Koornneef, J. 1983, A\&A, 128, 84

Kormendy, J., Fisher, D. B., Cornell, M. E., \& Bender, R. 2009, ApJS, 182, 216

Lauer, T. R., Gebhardt, K., Faber, S. M., et al. 2007, ApJ, 664, 226

Leja, J., Johnson, B. D., Conroy, C., \& van Dokkum, P. 2018, ApJ, 854, 62

Marconi, A., Risaliti, G., Gilli, R., et al. 2004, MNRAS, 351, 169

Müller Sánchez, F., Davies, R. I., Genzel, R., et al. 2009, ApJ, 691, 749

Mushotzky, R. F., Shimizu, T. T., Meléndez, M., \& Koss, M. 2014, ApJ, 781, L34

Nenkova, M., Ivezić, Ž., \& Elitzur, M. 2002, ApJ, 570, L9

Preto, M. 2010, GW Notes, 3, 3

Prieto, M. A., Marco, O., \& Gallimore, J. 2005, MNRAS, 364, L28

Raban, D., Jaffe, W., Röttgering, H., Meisenheimer, K., \& Tristram, K. R. W. 2009, MNRAS, 394, 1325

Rouan, D., Lacombe, F., Gendron, E., et al. 2004, A\&A, 417, L1

Santos, J. F. C., Dottori, H., \& Grosbø1, P. 2013, A\&A, 553, A74

Schodel, R., Gallego Cano, E., Nogueras Lara, F., Dong, H., \& Gallego Calvente, T. 2017, in Highlights on Spanish Astrophysics IX, eds. S. Arribas, A. Alonso-Herrero, F. Figueras, et al., 308

Thatte, N., Quirrenbach, A., Genzel, R., Maiolino, R., \& Tecza, M. 1997, ApJ, 490, 238

Young, S., Hough, J. H., Axon, D. J., Bailey, J. A., \& Ward, M. J. 1995, MNRAS, 272, 513 